\title{
Molecular interpretation of nonclassical gas dynamics of dense vapors under the van der Waals model
}

\author{
P. Colonna ${ }^{a)}$ \\ Energy Technology Section, Process and Energy Department, Delft University of Technology, \\ Leeghwaterstraat 44, 2628 CA Delft, The Netherlands \\ A. Guardone $e^{\text {b) }}$ \\ Dipartimento di Ingegneria Aerospaziale, Politecnico di Milano, Via La Masa 34, 20154 Milano, Italy
}

(Received 28 June 2005; accepted 23 March 2006; published online 16 May 2006)

\begin{abstract}
The van der Waals polytropic gas model is used to investigate the role of attractive and repulsive intermolecular forces and the influence of molecular complexity on the possible nonclassical gas dynamic behavior of vapors near the liquid-vapor saturation curve. The decrease of the sound speed upon isothermal compression is due to the well-known action of the van der Waals attractive forces and this effect is shown here to be comparatively larger for more complex molecules with a large number of active vibrational modes; for these fluids isentropic flows are in fact almost isothermal. Contributions to the speed of sound resulting from intermolecular forces and the role of molecular complexity are analyzed in details for both isothermal and isentropic transformations. Results of the exact solution to the problem of a finite pressure perturbation traveling in a still fluid are presented in three exemplary cases: ideal gas, dense gas and nonclassical gas behavior. A classification scheme of fluids based on the possibility of exhibiting different gas dynamic behaviors is also proposed.

(C) 2006 American Institute of Physics. [DOI: 10.1063/1.2196095]
\end{abstract}

\section{INTRODUCTION}

The theoretical and numerical study of nonclassical gas dynamics is an active research field. Recent advancements (see, e.g., Menikoff and Plhor, ${ }^{1}$ Cramer, ${ }^{2}$ and Kluwick ${ }^{3}$ for reviews) sparked some new attempts to experimentally verify the most dramatic of the predicted unconventional gas dynamic phenomena in a single-phase dense vapor, namely the existence of rarefaction shock waves (see, e.g., Refs. 4-7). A wealth of literature also reports analytical and numerical results regarding many different unconventional gas dynamic effects. Nozzle and simple geometries are treated in Refs. 8-12. Shock tube flows are treated, e.g., in Refs. 13-15. Two-dimensional flows exhibiting nonclassical effects through turbine cascades were simulated in Refs. 16-18.

As a preliminary introduction to the present study, the basic elements of the theory of shock formation in a nonideal compressible gas are briefly recalled, see, e.g., Hayes ${ }^{19}$ and Thompson. ${ }^{20}$ Figure 1 illustrates the propagation of a compression-rarefaction simple wave in a compressible medium. Denoting by $w$ the (local) speed of propagation of a weak pressure disturbance, one has $w=u+c$, being $u$ the local fluid velocity and $c$ the local speed of sound defined as

$$
c^{2} \equiv-v^{2}\left(\frac{\partial P}{\partial v}\right)_{s}
$$

where $v$ and $s$ are the specific volume and entropy, respectively, and $P$ is the pressure. From the theory of characteris-

\footnotetext{
${ }^{a)}$ Electronic mail: P.Colonna@3mE.TUDelft.nl

${ }^{b}$ Electronic mail: guardone@aero.polimi.it
}

tics, see, e.g., Ref. 21, along each characteristic curve $\mathcal{C}^{-}$the Riemann invariant $\mathcal{R}^{-}=u-F$, with

$$
F=F(P ; \bar{s})=\int_{P_{\mathrm{ref}}}^{P} \frac{v(\pi ; \bar{s})}{c(\pi ; \bar{s})} d \pi,
$$

is constant and therefore $d u=d F$ in the whole flow field. Substituting $d u=d F=(v / c) d P$ from (1) and $d c$ $=(\partial c / \partial P)_{s} d P=-(v / c)^{2}(\partial c / \partial v)_{s} d P$ in $d w=d u+d c$, yields ${ }^{22}$

$$
d w=\left[\frac{v}{c}-\frac{v^{2}}{c^{2}}\left(\frac{\partial c}{\partial v}\right)\right]_{s} d P=\frac{v}{c} \Gamma d P
$$

where $\Gamma$ is the fundamental derivative of gas dynamics ${ }^{23} \mathrm{de}-$ fined as

$$
\Gamma \equiv 1-\frac{v}{c}\left(\frac{\partial c}{\partial v}\right)_{s} .
$$

Equation (2) shows that compression waves $(d P>0)$ steepen $(d w>0)$ to form a compression shock only if $\Gamma>0$. This is indeed the case for a perfect or polytropic ideal gas, i.e., an ideal gas with constant isochoric specific heat. For an ideal gas $\Gamma=(\gamma+1) / 2=$ const $>1$, where $\gamma=c_{P} / c_{v}>1$ is the ratio of the (constant) isobaric and isochoric specific heats $c_{P}$ and $c_{v}$, respectively. Conversely, rarefaction waves $(d P<0)$ eventually form a rarefaction shock only if $\Gamma<0$. In the special case $\Gamma=0, d w \equiv 0$ across both compression and rarefaction waves, and the wave profile propagates in time with no distortions. These cases are summarized in Table I and sketched in Fig. 2.

Nonclassical wavefields can therefore occur only provided that the fundamental derivative of gas dynamics $\Gamma$ becomes negative. In addition, situations more complex than those depicted in Fig. 2 are possible, with $\Gamma$ changing sign 


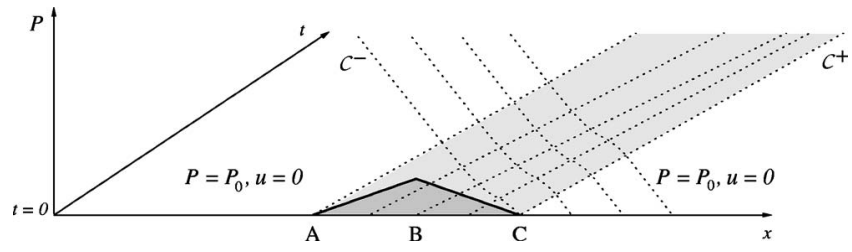

FIG. 1. Propagation of a right-running one-dimensional compressionrarefaction profile. Initial conditions at time $t=0$ (dark gray) in the $x$ - $P$ plane and representative characteristic lines of the $\mathcal{C}^{+}$and $\mathcal{C}^{-}$in the $x$ - $t$ plane. Shading (light gray) in the $x$ - $t$ plane indicates the nonuniform flow region.

during the flow evolution, thus leading to a wealth of different gas dynamic flow fields, including mixed nonclassical waves, split shocks and double sonic shocks, as reviewed, e.g., by Menikoff and Plhor. ${ }^{1}$ A detailed description of wave propagation in vapors exhibiting both positive and negative $\Gamma$ regions, including the effects of viscosity and thermal conductivity, is given by Cramer and Kluwick. ${ }^{25}$

Although experimental evidence of nonclassical gas dynamics in two-phase vapor/liquid ${ }^{26-30}$ or solid/solid ${ }^{31}$ systems has been documented in the past decades, no experimental proof of nonclassical phenomena in the vapor phase is currently available, with the only exception of the experiment of Borisov et al. ${ }^{5}$ in 1983, see also Kutateladze et al. ${ }^{32}$ Yet the interpretation of the results presented in Refs. 5 and 32 has been challenged by Cramer, ${ }^{33}$ Thompson, ${ }^{29}$ Fergason et al. $^{34,35}$ and the observed wavefield is now believed to be related to both critical point and two-phase nonclassical effects.

Fluids that are believed to exhibit a negative $\Gamma$ region in the vapor phase are also known as BZT vapors from Bethe, Zel'dovich, and Thompson who pioneered the subject of nonclassical gas dynamics theory. ${ }^{20,36,37}$

Many authors employed various equations of state with different levels of complexity and accuracy to demonstrate that, for some high molecular weight fluids, there exists a region at high reduced temperature and pressure close to saturation in which $\Gamma$ becomes negative, see, e.g., Refs. 22, 33, and 38-40. Examples are given by the fluids selected for experimental scrutiny of rarefaction shocks in the vapor phase and include hydrocarbons, per-fluorocarbons, ${ }^{34,35}$ and more recently siloxanes. ${ }^{12,39,41}$ In the cited references it is noted that, by substituting the definition of the speed of sound into (3), one also has

TABLE I. The classical and nonclassical wave character for different values of the fundamental derivative of gas dynamics $\Gamma$, see, e.g., Ref. 24.

\begin{tabular}{lllll}
\hline \hline$\Gamma>0$ & $d P>0$ & $\Rightarrow$ & $d w>0$ & Compression shock \\
& $d P<0$ & $\Rightarrow$ & $d w<0$ & Rarefaction (isentropic) wave \\
$\Gamma=0$ & $d P \lessgtr 0$ & $\Rightarrow$ & $d w=0$ & Stationary wave profiles \\
$\Gamma<0$ & $d P>0$ & $\Rightarrow$ & $d w<0$ & Compression (isentropic) wave \\
& $d P<0$ & $\Rightarrow$ & $d w>0$ & Rarefaction shock \\
\hline \hline
\end{tabular}

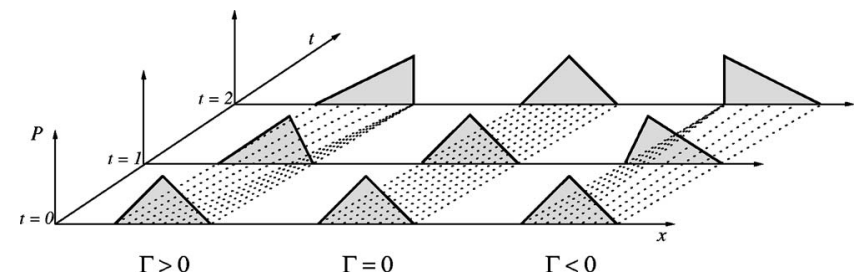

FIG. 2. Evolution of a compression-rarefaction profile for $\Gamma>0$ (left), $\Gamma$ $=0$ (center) and $\Gamma<0$ (right), from Ref. 20. Dotted lines indicate characteristic lines belonging to the $\mathcal{C}^{+}$family in the $x$ - $t$ plane. Pressure $P$ is the gauge pressure with respect to pressure $P_{0}$; the fluid velocity is zero where $P=P_{0}$.

$$
\Gamma=\frac{v^{3}}{2 c^{2}}\left(\frac{\partial^{2} P}{\partial v^{2}}\right)_{s}
$$

and therefore $\Gamma$ is negative if the isentropes in the volumepressure plane are concave down. This situation is reversed with respect to the well know ideal polytropic gas case, as shown in Fig. 3 where the region of negative $\Gamma$ and $\left(\partial^{2} P / \partial v^{2}\right)_{s}$ is illustrated for $c_{v} / R=50$.

The argument usually presented to provide a physical explanation to the possibility of observing nonclassical gas dynamic behavior in the vapor phase is as follows. In a region close to the liquid-vapor critical point and for volumes higher than the critical one, the isotherm in the $v-P$ plane presents a downward curvature (see, e.g., the critical isotherm in Fig. 3). In fluids with high isochoric specific heat, an isentropic transformation is almost isothermal, since from thermodynamics one has

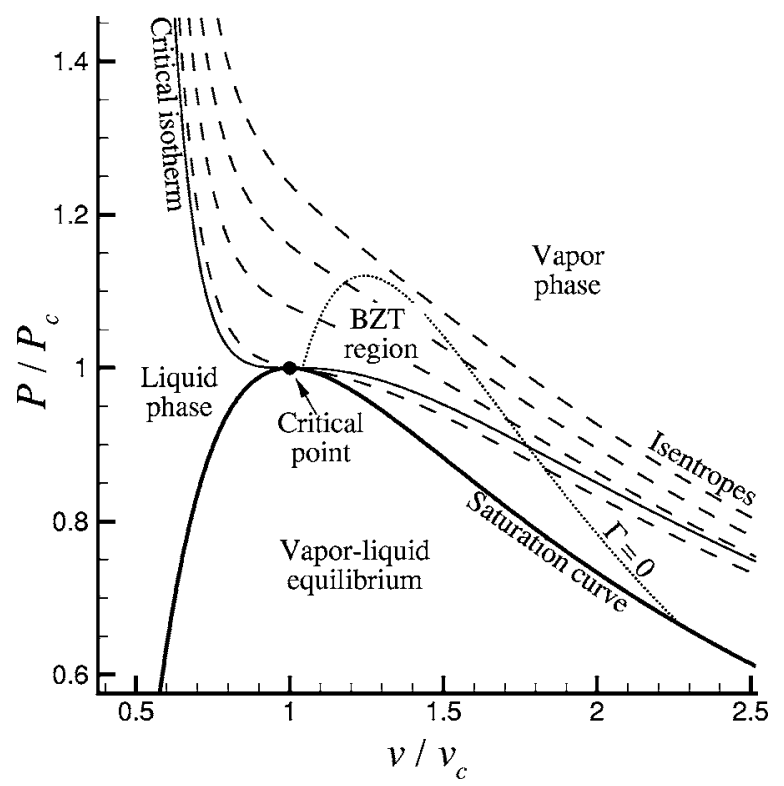

FIG. 3. The negative $\Gamma$ region (BZT region) in the reduced pressure-reduced specific volume thermodynamic plane for a polytropic van der Waals fluid with $c_{v} / R=50$. Several isentropes are also indicated as dashed lines and they highlight that the BZT region corresponds to the region in which they are curved downward, i.e., $\left(\partial^{2} P / \partial v^{2}\right)_{s}<0$. 
TABLE II. Relation between the fundamental derivative of gas dynamics $\Gamma$ and the speed of sound derivative along the isentropes, with corresponding classifications of the gas dynamic flow field (Ref. 20). The conditions $(\partial c / \partial v)_{s}>0$, which can be verified only in flows of nonideal gases, is necessary but not sufficient for nonclassical flow fields to be possible.

\begin{tabular}{ccr}
$\Gamma>1$ & $(\partial c / \partial v)_{s}<0$ & Classical (ideal gas) behavior \\
$0<\Gamma<1$ & $0<(\partial c / \partial v)_{s}<c / v$ & Classical (nonideal gas) behavior \\
$\Gamma<0$ & $(\partial c / \partial v)_{s}>c / v$ & Nonclassical behavior \\
\hline
\end{tabular}

$$
\left(\frac{\partial T}{\partial v}\right)_{s}=-\frac{T}{c_{v}}\left(\frac{\partial P}{\partial T}\right)_{v}
$$

Therefore, the higher the $c_{v}$, the more the isentropes will approach the isotherms, to eventually coincide with the latter in the limit $c_{v} \rightarrow \infty$. The negative curvature of the isentropic curves is therefore as that of the isotherms. This explanation is termed in the following as the thermodynamic or phenomenological interpretation. In Refs. 22 and 40, the thermodynamic explanation is extended to include other macroscopic properties, such as different models for a temperaturedependent $c_{v}$, the critical compressibility factor $Z_{\mathrm{c}}$, the Reidel parameter $\alpha$, and the acentric factor $\omega$, in addition to the constant isochoric heat capacity considered in earlier works.

In the present study, the attention is shifted from the thermodynamic viewpoint to the molecular or microscopic one, to elaborate an explanation directly in terms of the basic molecular characteristics, including vibrational degrees of freedom and intermolecular attractive/repulsive forces. To this purpose, the simple van der Waals gas model, which is characterized by a direct relation between the equation of state parameters and the molecular features, is used to elucidate the physical mechanism acting at molecular level that is responsible for the inversion of the gas dynamic behavior in compressible flows of high molecular weight fluids.

To conclude the introduction to shock wave formation in nonclassical flowfields, it should be noted that across a (right-running) rarefaction wave the fluid velocity always decreases $[d u=(v / c) d P<0]$ due to the action of the negative pressure difference across the wave; from (3), for $\Gamma$ to be negative and a rarefaction shock wave to be eventually formed $(d w>0)$, the speed of sound is therefore required to increase. In particular, $d c>|d u|$. Note that in a perfect gas, $c$ always decreases across a rarefaction wave. A nontrivial result from the thermodynamic theory of BZT vapors is that three different situations can possibly occur in the vapor phase. ${ }^{20}$ In the classical regime and for $\Gamma>1$, the speed of sound decreases across isentropic rarefactive processes and the well-known ideal gas dynamic behavior of compression shocks and isentropic rarefaction wave is encountered. For $\Gamma<0$, nonclassical rarefaction shocks or isentropic compression wave are predicted. In between, therefore for $0<\Gamma<1$, there exists an intermediate region in which only classical gas dynamic phenomena are possible, but the speed of sound dependence on the density is reversed with respect to that of an ideal gas. In Table II, the relation between the sound speed derivative and $\Gamma$ is summarized.
Not surprisingly, the peculiar behavior of the speed of sound in a real gas plays a major role in the determination of its nonclassical gas dynamic character. The first part of this work is therefore devoted to the study of the speed of sound in the vapor phase and to investigate the relation of the speed of sound and of its derivative with respect to density with parameters describing molecular features. To this purpose, isothermal transformations are first considered because the role of molecular parameters can easily be put into evidence. The limiting case of molecules with a very large number of available degrees of freedom provides the bridge connecting isothermal to isentropic transformations. Finally, some relevant behaviors with respect to the propagation of finite perturbations are classified and the corresponding analytical solutions are presented. A classification of vapors based on the possibility of displaying some or all the possible gas dynamic behaviors is also proposed.

\section{THE POLYTROPIC VAN DER WAALS MODEL AND MOLECULAR CHARACTERISTICS}

The pressure and internal energy equations of state (EOS) as originally stated by van der Waals are

$$
\begin{aligned}
& P(T, v)=\frac{R T}{v-b}-\frac{a}{v^{2}}, \\
& e(T, v)=c_{v} T-\frac{a}{v} .
\end{aligned}
$$

In the above equations $R, R=\mathcal{R} / M$, is the gas constant, with $M$ as the molecular weight and $\mathcal{R}=8.314 \mathrm{~J} /(\mathrm{mol} \mathrm{K})$ universal gas constant, $a$ is a gas-dependent parameter related to long range attractive intermolecular forces, $b$ is related to short range repulsive intermolecular forces, and $c_{v}$ is the isochoric specific heat, which is constant under the polytropic assumption considered here. Note that in the case of the van der Waals EOS $c_{v} \equiv(\partial e / \partial T)_{v}$ is a function of the temperature only and therefore, for asymptotic consistency with the dilute gas conditions, the function $c_{v}=c_{v}(T)$ is the same in the ideal gas state and in dense states.

By imposing the stability conditions at the critical point, i.e., $(\partial P / \partial v)_{T}=0$ and $\left(\partial^{2} P / \partial v^{2}\right)_{T}=0$, the fluid-dependent parameters $a$ and $b$ in the equations of state can be expressed in terms of the gas constant $R$ and the critical pressure $P_{\mathrm{c}}$ and temperature $T_{\mathrm{c}}$ as follows:

$$
a=\frac{27}{64} \frac{R^{2} T_{c}^{2}}{P_{c}}, \quad b=\frac{1}{8} \frac{R T_{c}}{P_{c}} .
$$

The van der Waals equation of state can be rigorously derived using a statistical mechanics approach from an intermolecular potential having in fact a repulsive and an attractive contribution (see, e.g., Ref. 42).

The polytropic assumption implies that $c_{v}$ is constant. The correctness of such an approximation in the evaluation of thermodynamic properties of dense gases has been already discussed in Refs. 22 and 40. This approximation is justified in the present study, given the small range of temperatures in which $\Gamma$ is negative. As a quantitative example, if one considers a rarefaction shock occurring in a PP10 [Pf- 
TABLE III. Molecular weight $M$, critical pressure $P_{c}$ and temperature $T_{c}$, dimensionless reciprocal specific heat $\delta=R / c_{v}$ evaluated at the critical temperature, active degrees of freedom $N=2 / \delta=2 c_{v} / R$, van der Waals coefficients $A$ and $B$ (per unit mole) and $a$ and $b$ (per unit mass) for several fluids.

\begin{tabular}{|c|c|c|c|c|c|c|c|c|c|}
\hline Fluid & $\begin{array}{c}M \\
{[\mathrm{~g} / \mathrm{mol}]}\end{array}$ & $\begin{array}{c}P_{c} \\
{[\mathrm{bar}]}\end{array}$ & $\begin{array}{c}T_{c} \\
{[\mathrm{~K}]}\end{array}$ & $\delta$ & $N$ & $\begin{array}{c}A \\
{\left[\mathrm{~Pa} \mathrm{~m}^{6} / \mathrm{mol}^{2}\right]}\end{array}$ & $\begin{array}{c}B \\
{\left[10^{5} \mathrm{~m}^{3} / \mathrm{mol}\right]}\end{array}$ & $\begin{array}{c}a \\
{\left[\mathrm{~Pa} \mathrm{~m}^{6} / 10^{5} \mathrm{~kg}^{2}\right]}\end{array}$ & $\begin{array}{c}b \\
{\left[\mathrm{~m}^{3} / \mathrm{kg}\right]}\end{array}$ \\
\hline $\mathrm{H}_{2}$ & 2.02 & 13.0 & 33.2 & 0.4329 & 4.6 & 0.025 & 2.65 & 605.9 & 1313.9 \\
\hline $\mathrm{He}$ & 4.00 & 2.3 & 5.2 & 0.6671 & 3.0 & 0.003 & 2.35 & 21.4 & 587.4 \\
\hline $\mathrm{CH}_{4}$ & 16.04 & 46.1 & 190.6 & 0.3309 & 6.0 & 0.230 & 4.30 & 89.3 & 267.9 \\
\hline $\mathrm{H}_{2} \mathrm{O}$ & 18.02 & 220.6 & 647.1 & 0.2908 & 3.9 & 0.554 & 3.05 & 170.5 & 169.1 \\
\hline $\mathrm{N}_{2}$ & 28.01 & 34.0 & 126.2 & 0.3862 & 5.2 & 0.137 & 3.86 & 17.4 & 137.7 \\
\hline $\mathrm{O}_{2}$ & 32.00 & 50.4 & 154.6 & 0.4121 & 4.9 & 0.138 & 3.19 & 13.5 & 99.6 \\
\hline $\mathrm{CO}_{2}$ & 44.01 & 73.8 & 304.2 & 0.2855 & 7.0 & 0.366 & 4.28 & 18.9 & 97.3 \\
\hline Toluene & 92.14 & 39.9 & 591.8 & 0.0452 & 44.3 & 2.558 & 15.40 & 30.1 & 167.3 \\
\hline Octane & 114.20 & 24.3 & 568.8 & 0.0273 & 73.3 & 3.886 & 24.35 & 29.8 & 213.0 \\
\hline Decane & 142.29 & 20.6 & 617.7 & 0.0205 & 97.5 & 5.388 & 31.09 & 26.7 & 219.0 \\
\hline Dodecane & 170.34 & 17.7 & 658.2 & 0.0164 & 121.6 & 7.127 & 38.59 & 24.6 & 226.9 \\
\hline D4 & 296.62 & 13.3 & 586.5 & 0.0157 & 127.6 & 7.531 & 45.76 & 8.6 & 154.5 \\
\hline PP5 & 462.00 & 17.1 & 565.2 & 0.0156 & 128.5 & 5.455 & 34.40 & 2.5 & 74.3 \\
\hline PP10 & 574.00 & 15.8 & 632.2 & 0.0128 & 156.2 & 7.394 & 41.68 & 2.2 & 72.5 \\
\hline
\end{tabular}

perhydrofluorene, $\mathrm{C}_{13} \mathrm{~F}_{22}$ ] flow entirely enclosed in the negative $\Gamma$ region, the corresponding temperature decrease is of about $3 \mathrm{~K}^{43}$ According to the simplified nonpolytropic model considered in Ref. 43, this causes a change of about $0.23 \%$ in the value of the specific heat.

Attractive forces, repulsive forces, and heat capacity are related in a complicated way to molecular complexity. Table III shows the values of $a$ and $b$ and $\delta=R / c_{v}\left(T_{c}\right)$, for several fluids listed in order of increasing molecular weight. The coefficients $a$ and $b$ provide a measure of the strength of intermolecular forces per unit mass and therefore their value depends on the molecular weight. To appreciate the relation between molecular complexity and attractive and repulsive forces acting on a single molecule, the following coefficient per unit mole

$$
A=a \cdot M^{2} \quad \text { and } \quad B=b \cdot M
$$

must be considered: values of $A$ and $B$ are also reported in Table III. From the table it is therefore apparent that the heavier the molecule, the greater attractive and repulsive intermolecular forces tend to be. Note that the increase in the molecular weight $M$ more than overcome the increase of intermolecular forces $(A$ and $B)$ acting on each molecule and therefore the mass-specific van der Waals parameters $a$ $=A / M$ and $b / M$ are found to decrease with increasing molecular weight.

For the following treatment it is useful to consider the van der Waals equation of state in the nondimensional form

$$
P_{r}\left(T_{r}, v_{r}\right)=\frac{8 T_{r}}{3 v_{r}-1}-\frac{3}{v_{r}^{2}}
$$

In the above equation $P_{r}, T_{r}$ and $v_{r}$ are, respectively, the reduced pressure, temperature, and specific volume, defined as

$$
T_{r}=\frac{T}{T_{c}}, \quad P_{r}=\frac{P}{P_{c}} \quad \text { and } v_{r}=\frac{v}{v_{c}},
$$

with $v_{c}$ specific volume at the critical point. Correspondingly, the dimensionless EOS parameters $a$ and $b$ read

$$
a_{r}=\frac{a}{P_{c} v_{c}^{2}}=3 \quad \text { and } \quad b_{r}=\frac{b}{v_{c}}=\frac{1}{3} .
$$

Under the van der Waals approximation therefore the attractive and repulsive force parameters in the reduced form do not depend on the fluid under consideration.

The compatible equation for the internal energy in terms of reduced variables reads

$$
e_{r}\left(T_{r}, v_{r}\right)=\frac{8}{3} \frac{T_{r}}{\delta}-\frac{3}{v_{r}},
$$

where $e_{r}=e /\left(P_{c} v_{c}\right)$ and $\delta=R / c_{v}$. Therefore, the complete polytropic van der Waals model in reduced form depends only on the value of the dimensionless reciprocal specific heat $\delta$.

The value of $\delta$ is related to the number $N$ of active degrees of freedom of a molecule. Considering only the translational, rotational and vibrational degrees of freedom, the number $N^{\text {avail. }}$ of available degrees of freedom is a linear function of the number of atoms $Q$ constituting the molecule, namely, $N^{\text {avail. }}=6 Q-5-\varepsilon$, with $\varepsilon=-2,0,1$ for monatomic, linear polyatomic and nonlinear polyatomic molecules, respectively. At a given temperature, $N$ can be lower than $N^{\text {avail. }}$, i.e., not all the available modes are activated, see, e.g., Ref. 44.

According to the energy equipartition principle, ${ }^{44}$ at a given temperature, each fully activated degree of freedom contributes $R / 2$ to the overall value of the isochoric specific heat, so that $c_{v}=N \mathcal{R} /(2 M)$ or, from the definition of $\delta$, 


$$
\delta=\frac{2}{N}
$$

It is worthwhile pointing out that, because at a given temperature vibrational degrees of freedom can be partially activated, $N$ can also assume noninteger values. Moreover, $\delta$ does not depend on the molecular weight $M$ but only on the number of active degrees of freedom of the molecules, namely, on the complexity of the molecule.

The above discussion leads us to define molecular complexity as the number of the active degrees of freedom of a molecule. For a polytropic van der Waals fluid, $N($ or $\delta$ ) is the only parameter that is available to distinguish among different substances. The classification of BZT fluids using the molecular complexity $N$, instead of the value of $c_{v}$ or $M$ as it is standard practice in the literature, is introduced here in view of the use of more complex gas models that include additional molecular parameters to represent the thermodynamic behavior.

It is important to observe from Table III that the molecular weight is directly related to the molecular complexity for fluids belonging to the same class. However, lighter molecules can be more complex than heavier molecules, whenever they are formed by a larger number of atoms. Consider, e.g., methane $\left(\mathrm{CH}_{4}, M=16.04 \mathrm{~g} / \mathrm{mol}\right)$ and molecular oxygen $\left(\mathrm{O}_{2}, M=32.00 \mathrm{~g} / \mathrm{mol}\right)$. The number of atoms and hence $N^{\text {avail. }}$ is higher for methane, though its molecular weight is lower; correspondingly, the value of $\mathrm{N}$ for $\mathrm{CH}_{4}$ is higher than that for molecular oxygen. The same reasoning can be applied to PP10 and $\mathrm{D}_{4}$, two fluids taken into consideration for the experimental demonstration of BZT effects: PP10 has almost twice the molecular weight of $\mathrm{D}_{4}$ but the two molecules have almost the same degree of complexity leading to almost the same value of $N$.

\section{SPEED OF SOUND IN THE VAPOR PHASE}

The expression for the speed of sound as a function of the temperature and the specific volume can be obtained from its definition $c^{2}(s, v) \equiv-v^{2}(\partial P / \partial v)_{s}$ and from the van der Waals model (4) as

$$
c^{2}(T, v)=\left(1+\frac{2}{N}\right) R T\left[\frac{v}{v-b}\right]^{2}-\frac{2 a}{v} .
$$

In order to study the influence of molecular characteristics, it is convenient to express $c$ as the sum of the contributions from the ideal gas sound speed and of terms related to the effects of (both) van der Waals forces, namely

$$
c^{2}=c_{\text {ideal }}^{2}+\delta c_{\text {rep }}^{2}+\delta c_{\text {attr }}^{2}
$$

where the contributions of the ideal gas term and of the two van der Waals corrections accounting for repulsive and attractive forces are

$$
c_{\text {ideal }}^{2}(T) \equiv\left(1+\frac{2}{N}\right) R T>0,
$$

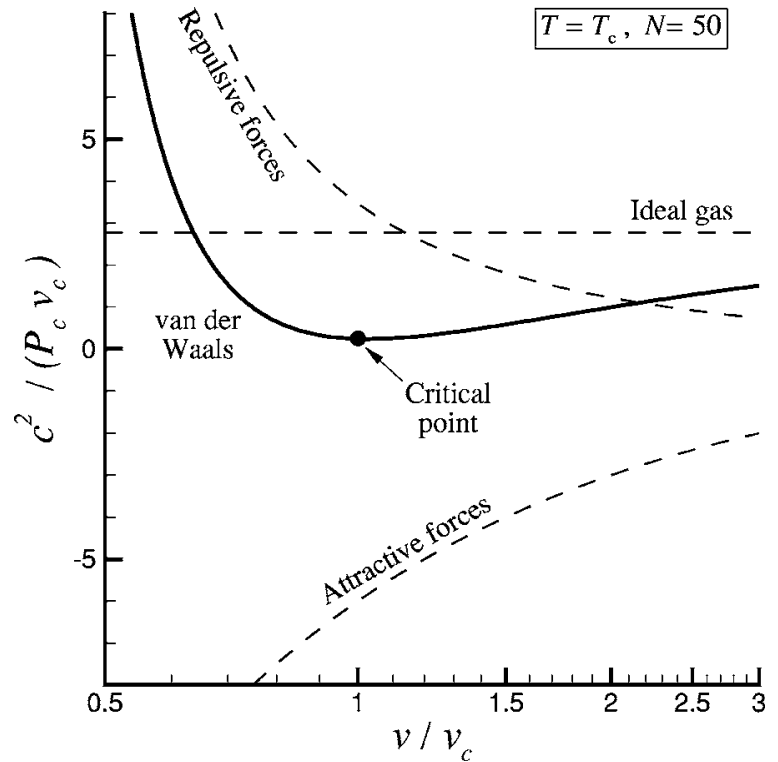

FIG. 4. Dimensionless speed of sound along the critical isotherm as a function of the reduced specific volume for the van der Waals gas and for the ideal gas for $N=50$. The dense gas contributions to the speed of sound as in relation (12) are also shown.

$$
\begin{aligned}
& \delta c_{\text {rep }}^{2}(T, v) \equiv\left(1+\frac{2}{N}\right) R T b \frac{2 v-b}{(v-b)^{2}} \\
&=c_{\text {ideal }}^{2}(T) b \frac{2 v-b}{(v-b)^{2}}>0, \\
& \delta c_{\text {attr }}^{2}(v) \equiv-\frac{2 a}{v}<0 .
\end{aligned}
$$

From the expressions above it can be noted that, differently from the dilute gas model, in which the sound speed is a function of the temperature only, the sound speed depends also on the specific volume if attractive and repulsive intermolecular forces are accounted for. The relative magnitude of the contributions to the dimensional speed of sound in Eq. (12) is illustrated in Fig. 4.

In particular, the different contributions to the value of $c$ can be discussed as follows:

Ideal gas contribution: From the viewpoint of the kinetic theory of gases, $c_{\text {ideal }}^{2}(T)$ in Eq. (12) is related to the molecular interaction associated with elastic collisions. Their effect increases with temperature because the average molecular speed scales with $\sqrt{T}$, and it is independent of $v$, in accordance with the ideal gas assumption of dimensionless (pointwise) molecules. Thus, $c_{\text {ideal }}^{2}(T)$ is constant along isotherms.

Influence of repulsive forces $(b)$ : From (12), the repulsive forces connected with the presence of the constant $b$ are seen to contribute positively to $c^{2}$ : at a given temperature and specific volume the speed of sound calculated by taking into account repulsive forces is higher with respect to that based on the ideal gas assumption. Moreover, as the specific volume tends to the molecular volume, this contribution tends to infinity. The limiting case $v_{r} \rightarrow b_{r}=\frac{1}{3}$, corresponds to the situation of highest possible density, namely, no further compression is possible. The compressibility of the substance is 
therefore zero and, correspondingly, the sound speed goes to infinity.

Influence of attractive forces (a): Attractive long range forces increase the compressibility of a fluid and therefore the speed of sound of a substance under the influence of attractive forces is lower than that of an ideal gas in the same thermodynamic state, see Eq. (12). As expected, the compression of a number of molecules is made easier if they are interacting attractively.

\section{Relation between molecular complexity and the sound speed value}

To analyze the influence of molecular complexity, it is more convenient to recast the expression (12) for the speed of sound in nondimensional form, namely,

$$
c_{r}^{2}=\frac{8}{3}\left(1+\frac{2}{N}\right)\left[\frac{3 v_{r}}{3 v_{r}-1}\right]^{2}-\frac{6}{v_{r}}<0,
$$

where $c_{r}^{2}=c^{2} / \sqrt{P_{c} v_{c}}$. The contributions stemming from the ideal gas behavior and the repulsive and attractive intermolecular forces read

$$
\begin{aligned}
& c_{r, \text { ideal }}^{2}\left(T_{r}\right)=\frac{8}{3}\left(1+\frac{2}{N}\right) T_{r}>0, \\
& \delta c_{r, \text { rep }}^{2}\left(T_{r}, v_{r}\right)=c_{\text {ideal }}^{2}\left(T_{r}, v_{r}\right) \frac{6 v_{r}-1}{\left(3 v_{r}-1\right)^{2}}>0, \\
& \delta c_{r, \text { attr }}^{2}\left(v_{r}\right)=-\frac{6}{v_{r}}<0 .
\end{aligned}
$$

The relations above allow to compare the properties of different substances at the same reduced conditions. The ideal gas and repulsive forces contributions include the factor 1 $+2 / N$ which reduces with increasing molecular complexity. The above is not surprising, since the ideal and repulsive contributions are connected with the internal energy of the translational modes, namely, to the average molecular speed squared; the more complex the molecule is, the more vibrational modes are available to store the energy, at the expenses of the translational terms. In other words, the energy transfer associated with the density perturbation is partly absorbed by the molecules, so that the more complex the molecule is, the greater is its capacity to absorb energy and the less energy is transferred to the translational modes which are responsible for sound propagation.

Figure 5 is useful to compare the influence of molecular complexity on the value of the sound speed for different fluids. In reduced terms the value of $c_{r}$ along the critical isotherm inversely depends on molecular complexity and for more complex molecules the decrease of $c$ with decreasing specific volume before critical conditions is seen to be stronger. Figure 6 shows that the same holds also for the dimensional quantities. In this respect, note that the reducing factor for the speed of sound is $\sqrt{P_{c} v_{c}}$ or $\sqrt{8 R T_{c} / 3}$ and that, at least for fluids belonging to the same family, both $R$ and $T_{c}$ usually increase with the molecular weight or complexity, see Table III. Correspondingly, the speed of sound at the same

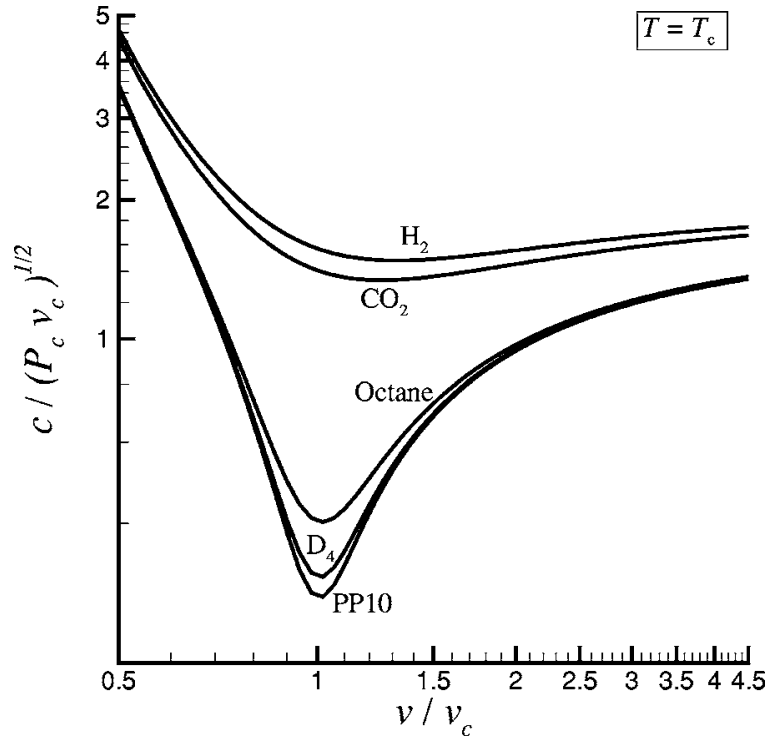

FIG. 5. Dimensionless speed of sound along the critical isotherm for fluids selected from Table III.

reduced conditions is lower for more complex molecules. It is worthwhile recalling that the polytropic van der Waals model fails to give an accurate representation of the thermodynamic properties in the dense vapor region but nevertheless the picture portrays faithfully the qualitative influence of molecular complexity on the sound speed.

\section{SPEED OF SOUND ALONG ISOTHERMAL TRANSFORMATIONS}

The existence of nonclassical gas dynamic phenomena is related to the dependence of the speed of sound $c$ on the specific volume and not on the absolute value of the speed of sound itself. The analysis of the derivative of $c$ is now performed in two steps. First, isothermal transformations are

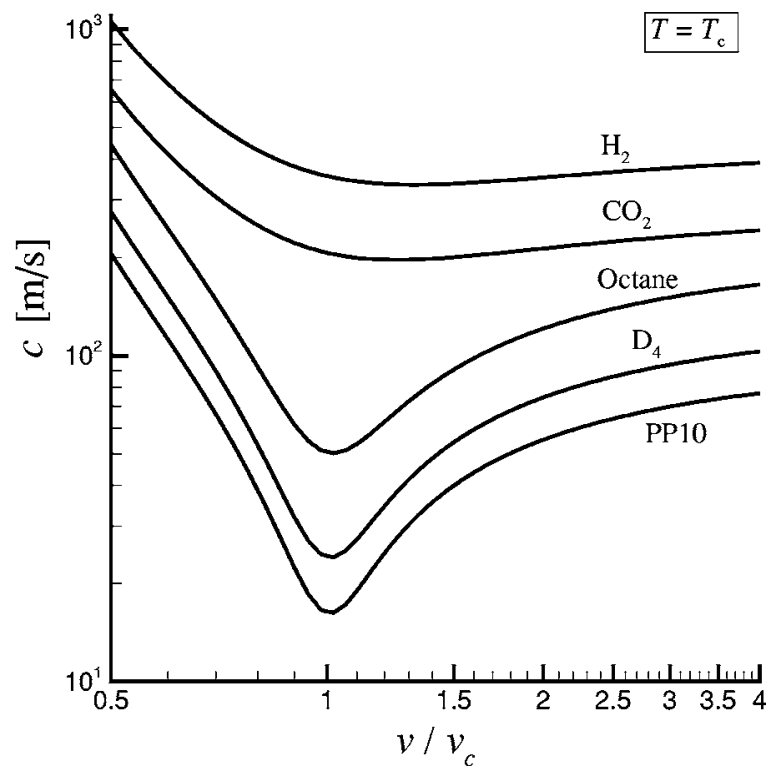

FIG. 6. Speed of sound along the critical isotherm for fluids selected from Table III, cf. Fig. 5. 


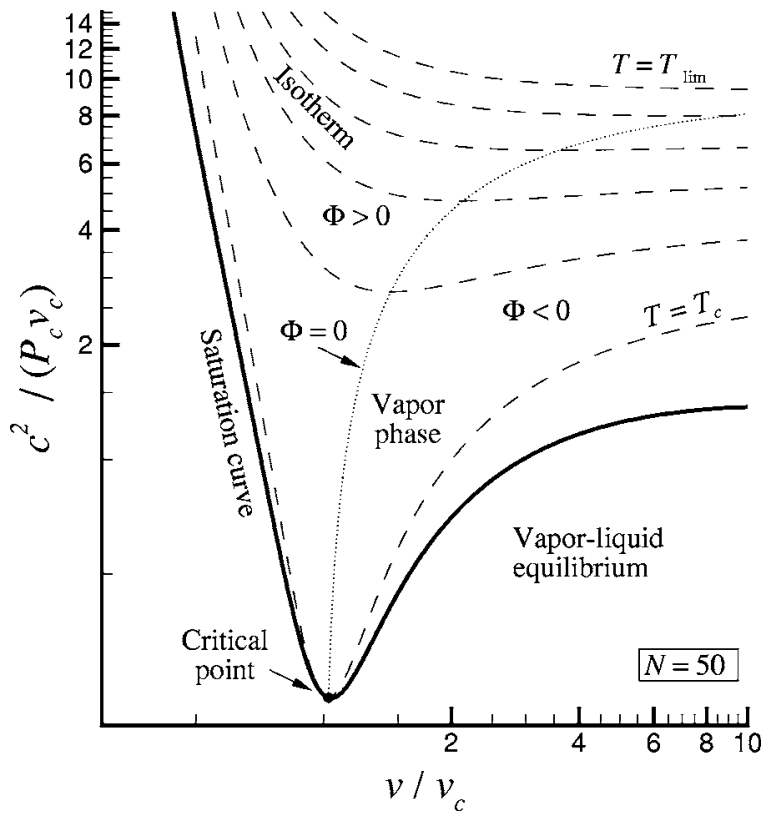

FIG. 7. Dimensionless speed of sound along supercritical isotherms and locus of the thermodynamic states in which $(\partial c / \partial v)_{T}=0(\Phi=0)$ for $N=50$. For $T \geqslant T_{\text {lim }}=(N /(N+2)) \frac{27}{8} T_{c}$, the sound speed is a monotone decreasing function of the specific volume. The saturation curve is also shown.

considered, for simplicity and to establish a direct connection with basic thermodynamic principles. Then, in the next section, isentropic compressions and expansions are addressed.

The variation of sound speed with the specific volume along the critical isotherm and the relative importance of the contributing terms are shown in Fig. 4 for a fluid with $N$ $=50$. The ideal gas contribution to the dimensionless speed of sound is constant and equal to 2.77. At $v_{r}=3$ for example, the repulsive forces contribute an additional 0.74, while the contribution of attractive forces is negative and equal to -2 ; the dimensionless van der Waals speed of sound at $v_{r}=3$ is therefore equal to 1.51 , i.e., almost half of the corresponding ideal-gas value. In the dense gas region $\left(1<v_{r} \lesssim 1.5\right)$ attractive forces dominate; at lower specific volumes, the increase in the strength of the repulsive forces is more than compensated by that of attractive ones and the speed of sound eventually decreases. For specific volumes lower than the critical one, the opposite is true, and the sound speed increases with decreasing specific volume. A minimum value for the speed of sound is therefore attained at the critical point, where the compressibility goes to infinity.

In order to further analyze the dependence of $c$ on $v$, it is useful to introduce the following dimensionless derivative

$$
\Phi \equiv-\frac{v}{c}\left(\frac{\partial c}{\partial v}\right)_{T}
$$

For a van der Waals fluid, a direct evaluation gives

$$
\Phi\left(T_{r}, v_{r}\right)=6 \frac{v_{r}}{c_{r}^{2}}\left[\left(4+\frac{8}{N}\right) \frac{T_{r} v_{r}}{\left(3 v_{r}-1\right)^{3}}-\frac{1}{2 v_{r}^{2}}\right] .
$$

The state of minimum sound speed is obtained from the condition $\Phi=0$.

Figure 7 shows that, for supercritical temperatures, the

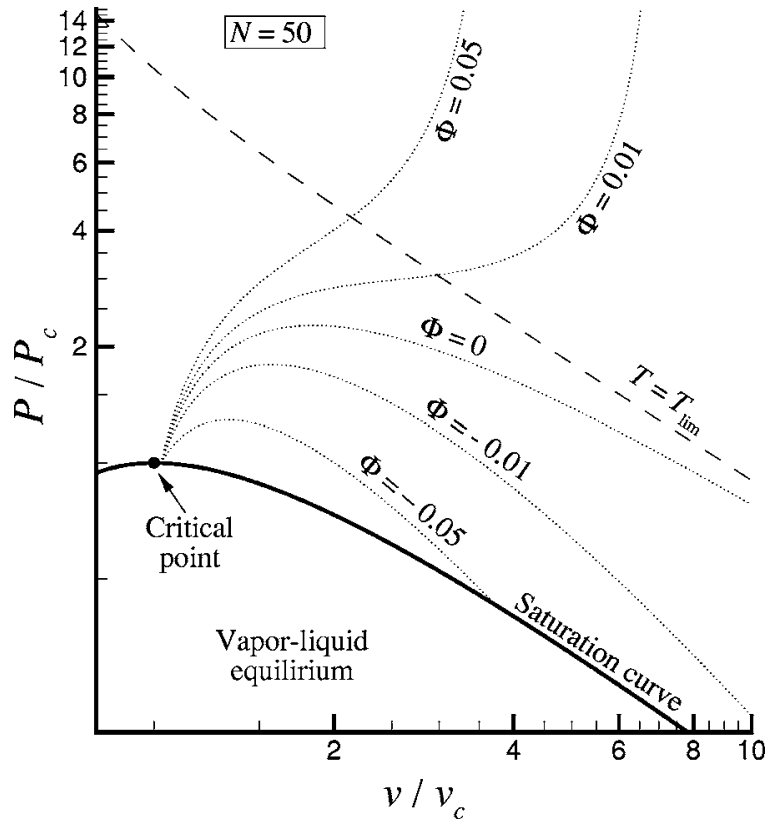

FIG. 8. Lines at constant $\Phi$ plane and the limiting isotherm $T_{\text {lim }}=(N /(N$ +2)) $\frac{27}{8} T_{c}$ in the $P_{r}-v_{r}$.

minimum value is attained at specific volumes higher than the critical value. More precisely, the higher the temperature, the higher the specific volume for the minimum sound speed, due to the higher value of the ideal gas and of the repulsive force contributions, see relations (14). Figure 8 shows several iso- $\Phi$ lines for a van der Waals fluid with $N=50$ and the limiting isotherm $T_{\lim }=(N /(N+2)) \frac{27}{8} T_{c}$ above which no minimum can be found. Figure 9 displays the loci of the

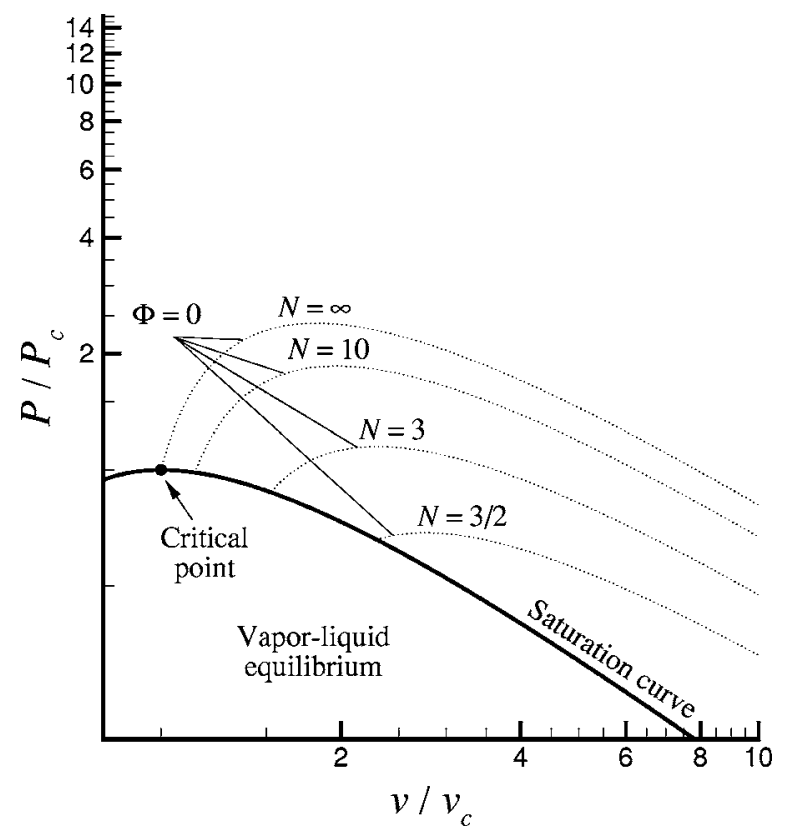

FIG. 9. Saturation curve and $\Phi=0$ curves for different values of the parameter $N$, ranging from $N=3$, corresponding to the lowest possible value of $N$ for monatomic gases, to the limit case, $N \rightarrow \infty$, corresponding to the idealized situation of molecules with an infinite number of internal degrees of freedom. In the regions where $\Phi<0$ the speed of sound decreases with decreasing $v$ along an isotherm, i.e., real vapor effects can manifest. 
minima of the speed of sound in the $P_{r}-v_{r}$ plane for several values of $N$. Since the saturation curve is independent of $N$, in Fig. 9 all possible van der Waals fluids are considered, from the simplest monatomic gas $(N=3)$ to the limiting case of molecules with an infinite number of internal degrees of freedom $(N \rightarrow \infty)$. For thermodynamic states above the $\Phi$ $=0$ line, $\Phi>0$ and the speed of sound increases on isothermal compressions; inside the $\Phi<0$ region, the opposite is true. Note that a region in which $\Phi<0$ exists for all possible van der Waals fluids and that the size of the $\Phi<0$ region increases with $N$.

As revealed by Eq. (16), the magnitude of the speed of sound variation with respect to the specific volume depends heavily on molecular complexity: in the $\Phi<0$ region, the greater $N$, the more negative is $\Phi$. In fact in a given reduced thermodynamic state, the positive contribution of the first term $(4+8 / N)\left(T_{r} v_{r} /\left(3 v_{r}-1\right)^{3}\right)$ decreases with $N$, while the second term, $1 / v_{r}^{2}$, remains constant. Moreover, the factor $6 v_{r} / c_{r}^{2}$ increases with decreasing $N$ because $c_{r}^{2}$ decreases with $N$.

It can be deduced therefore that more complex molecules tend to have smaller absolute values for the speed of sound in the same reduced state (see Sec. III), but greater variations of $c$ with density. This is due to the combined and mutual effect of repulsive and attractive forces together with the influence of molecular complexity. It is this strong variation of the speed of sound with density, although only along isentropic transformations, which determines the possibility of nonclassical phenomena in the vapor phase.

\section{SPEED OF SOUND ALONG ISENTROPIC TRANSFORMATIONS}

As outlined in the Introduction, the insights gained by relating the sound speed along isothermal transformations to molecular parameters can be extended to isentropic transformations. The bridge connecting isothermal and isentropic transformations is represented by the $N \rightarrow \infty$ limit, namely, the idealized case of a molecule with an infinite number of degrees of freedom. For $N \rightarrow \infty$ isentropic transformation are isothermal and therefore the explanations put forward in the previous section hold also for isentropic transformations. In fact, by recalling the thermodynamic identity

$$
\left(\frac{\partial T}{\partial v}\right)_{s}=-\frac{T}{c_{v}}\left(\frac{\partial P}{\partial T}\right)_{v}
$$

since thermodynamic stability implies $c_{v}>0$ and $(\partial P / \partial T)_{v}$ $>0$, the temperature is found always to decrease on isentropic expansion and to increase on isentropic compression for fluids with any finite value of $N$.

Beside the idealized case of molecules with an infinite number of atoms, it is interesting here to consider substances characterized by a high value of $N$, i.e., fluids made of complex molecules such as $\mathrm{D}_{4}$ [octamethylcyclotetrasiloxane, $\left(\left(\mathrm{CH}_{3}\right)_{2} \mathrm{SiO}\right)_{4}$ ] with $N=127.4$ or PP10 [Pf-perhydrofluorene, $\left.\mathrm{C}_{13} \mathrm{~F}_{22}\right]$ with $N=156.3$. In this case indeed along isentropic transformations the temperature variation is very limited and this allows us to extend the conceptual results established for isothermal transformations in the preceding section.
Similarly to the previous isothermal analysis, the contributions of the ideal gas term and of the repulsive and attractive forces can be written as functions of the reduced specific entropy $s_{r}$ and volume $v_{r}$ as

$$
\begin{aligned}
& c_{r, \text { ideal }}^{2}\left(s_{r}, v_{r}\right)=2 K_{0, r} \frac{N+2}{N^{2}} \frac{\exp \left(2 s_{r} / N\right)}{v_{r}^{2 / N}}>0, \\
& \delta c_{r, \text { rep }}^{2}\left(s_{r}, v_{r}\right)=c_{r, \text { ideal }}^{2}\left(s_{r}, v_{r}\right)\left[\left(\frac{3 v_{r}}{3 v_{r}-1}\right)^{2+2 / N}-1\right]>0,
\end{aligned}
$$

$$
\delta c_{r, \text { attr }}^{2}\left(v_{r}\right)=-\frac{6}{v_{r}}<0,
$$

where $s_{r}=s / R$ is the reduced entropy and $K_{0, r}$ is a constant. Note that the repulsive forces contribution is always positive, since $v_{r}>b / v_{c}=1 / 3$ and $N>0$. The sign of the three contributions are unchanged, with the ideal term and repulsive forces providing a positive contribution to the speed of sound while the attractive term is always negative.

To investigate the effect of molecular parameters on the variation of the sound speed along isentropes, the dimensionless derivative

$$
\Psi \equiv-\frac{v}{c}\left(\frac{\partial c}{\partial v}\right)_{s}
$$

can be analyzed. For a polytropic van der Waals gas the above expression becomes

$$
\Psi\left(T_{r}, v_{r}\right)=-54\left(1+\frac{2}{N}\right) \frac{T_{r} v_{r}}{\left(3 v_{r}-1\right)^{3}}\left(\frac{1}{N}+\frac{1}{3 v_{r}}\right)+\frac{6}{v_{r}},
$$

which is expressed here as a function of the reduced temperature $T_{r}$ and the specific volume $v_{r}$ to facilitate the comparison with its isothermal counterpart $\Phi$.

If $\Psi<0$, the sound speed decreases on isentropic compression, a necessary but not sufficient condition for nonclassical gas dynamic phenomena to occur. Following Thompson, ${ }^{24}$ the $\Psi=0$ locus is indicated hereinafter as the acoustic line; in fact, through isentropic compressions/ expansions occurring along the $\Psi=0$ line the speed of sound remains constant as in the acoustic approximation.

In the idealized case of $N \rightarrow \infty, \Psi \equiv \Phi$, and the $\Psi<0$ region in Fig. 10 is coincident with the $\Phi<0$ region in Fig. 9. The difference between the $\Phi<0$ and $\Psi<0$ regions augments as $N$ is decreased, as shown in Fig. 10.

The possibility for the sound speed to decrease on isothermal compression is related to the action of intermolecular attractive forces, with the molecular motion (ideal gas contribution) and the contribution of repulsive forces acting in the opposite direction. Both the ideal gas and repulsive terms are linearly increasing functions of the temperature [cf. Eq. (17)]. Across isothermal compressions or expansions, the ideal gas contribution to the speed of sound remains constant, whereas in isentropic transformations the $c_{\text {ideal }}^{2}$ term in Eq. (17) varies, since the specific volume varies. In particular, $c_{\text {ideal }}^{2}$ increases on compressions and decreases on 


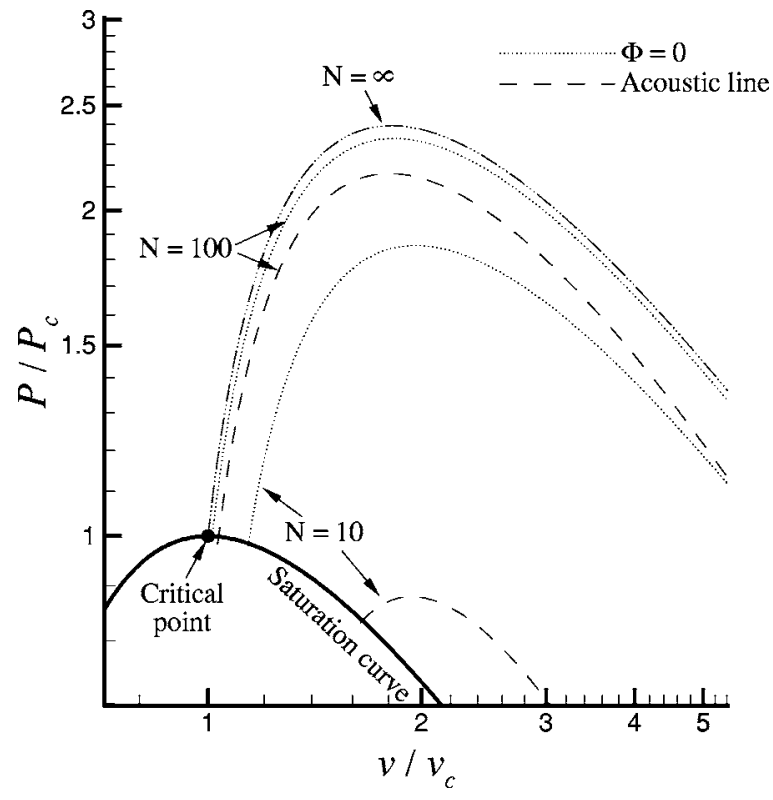

FIG. 10. $\Phi=0$ and $\Psi=0$ (acoustic line) loci for two different values of the molecular complexity $N$. As $N \rightarrow \infty$, the two curves become one and the same curve.

expansion. Note that $c_{\text {ideal }}^{2}$ appears also in the expression of the repulsive term $\delta c_{\text {rep }}^{2}$; correspondingly, changes in $\delta c_{\text {rep }}^{2}$ are amplified by the temperature difference across the wave in contrast with isothermal transformations. For a given fluid and thermodynamic state therefore $\Psi$ is always greater than $\Phi$.

Figure 11 shows that there exists a minimum value of $N$ allowing for the existence of a $\Psi<0$ region in the vapor phase. Vapors allowing for $\Psi<0$ regions are defined as high molecular complexity (HMC) fluids in the following, to un-

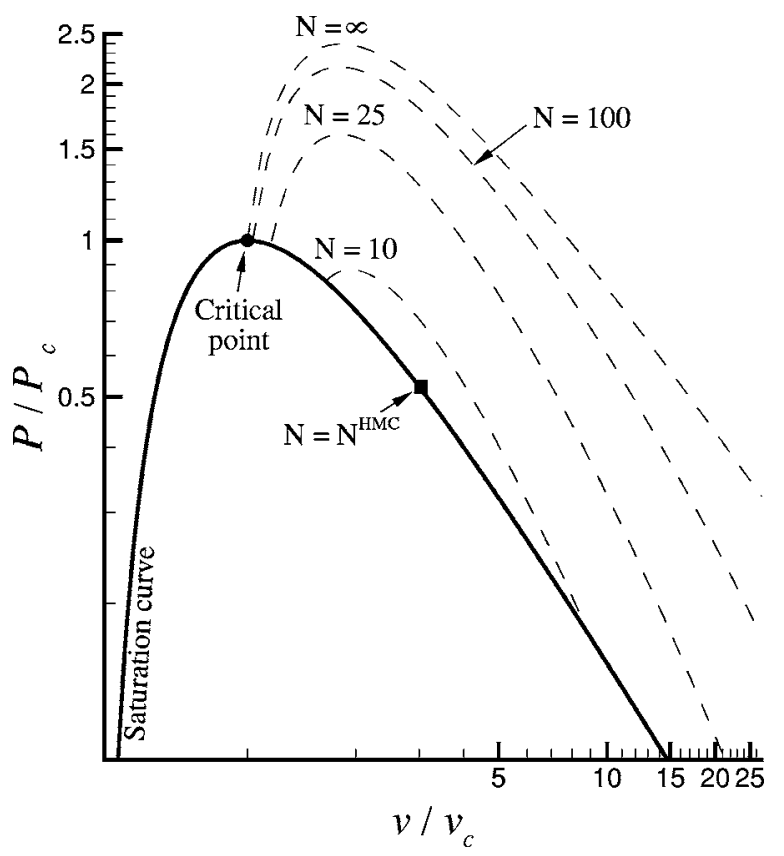

FIG. 11. Acoustic line $(\Psi=0)$ for different values of molecular complexity $N$. The minimum value of $N^{\mathrm{HWM}}=7.57$, for which a region of negative $\Psi$ exists is also indicated.

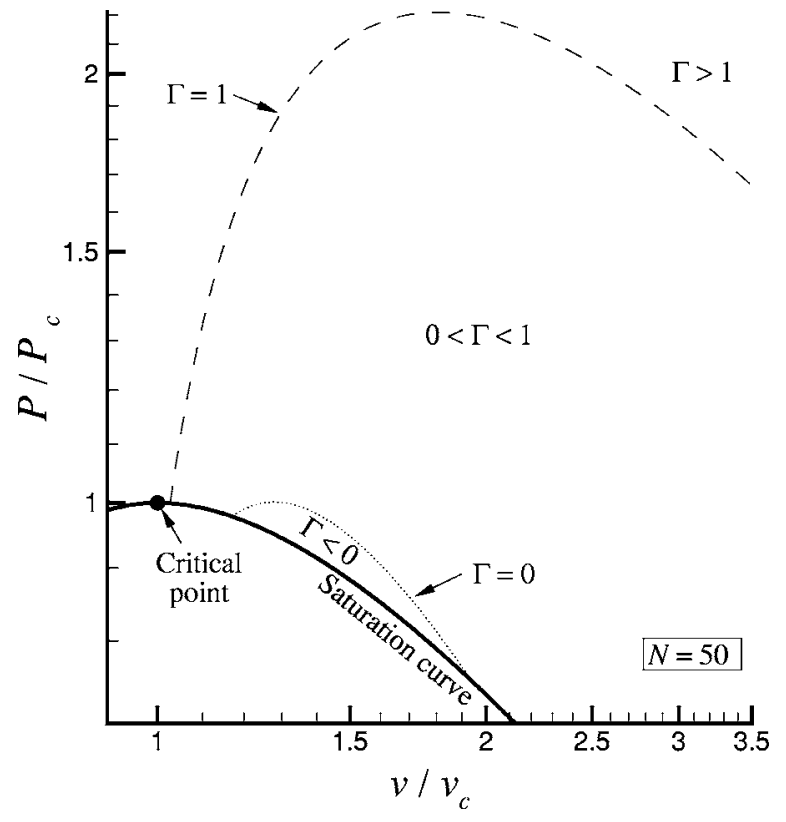

FIG. 12. Acoustic line ( $\Psi=0$, i.e., $\Gamma=1)$ and $\Gamma=0$ locus for a van der Waals fluid with $N=50$.

derline the fact that a necessary condition for $\Psi$ to be negative in the vapor phase is that the fluid molecules must be complex, i.e., the values of $N$ must be high $\left(N>N^{\mathrm{HMC}}\right.$ ح.57). This value has been found by Kluwick, ${ }^{45}$ who also showed a nonmonotone dependence of the speed of sound in isentropic expansions through nozzle for fluids with $N$ $>N^{\mathrm{HMC}} \simeq 7.57$. From Table III, for example, toluene and octane, together with heavier substances, are HMC fluids under the van der Waals model.

Nonclassical gas dynamics phenomena occur if the wave speed decreases across an isentropic compression wave. This happens if the reduction in the speed of sound is greater than the increase of the fluid velocity past the wave, i.e., $-d c$ $>d u . \Gamma<0$ is a more restrictive condition with respect to $\Psi<0$. In fact, from the definition of the fundamental derivative of gas dynamics (3)

$$
\Gamma=\Psi-1
$$

and therefore $\Gamma<\Psi, \Phi$. For a given fluid, i.e., for a given value of the parameter $N$, the $\Gamma<0$ region is therefore smaller than the $\Psi<0$, as depicted in Fig. 12.

The $\Gamma<0$ region, in which nonclassical gas dynamics phenomena are possible, is largest for $N \rightarrow \infty$; for smaller values of $N$, the size of the $\Gamma<0$ region reduces, as shown in Fig. 13. For $N=N^{\mathrm{BZT}} \simeq 33.33$, the $\Gamma=0$ line is tangent to the saturation curve; for $N<N^{\mathrm{BZT}}$, nonclassical gas dynamic behavior is forbidden, at least in the vapor phase.

The expression of the fundamental derivative of gas dynamics for a van der Waals fluid, 


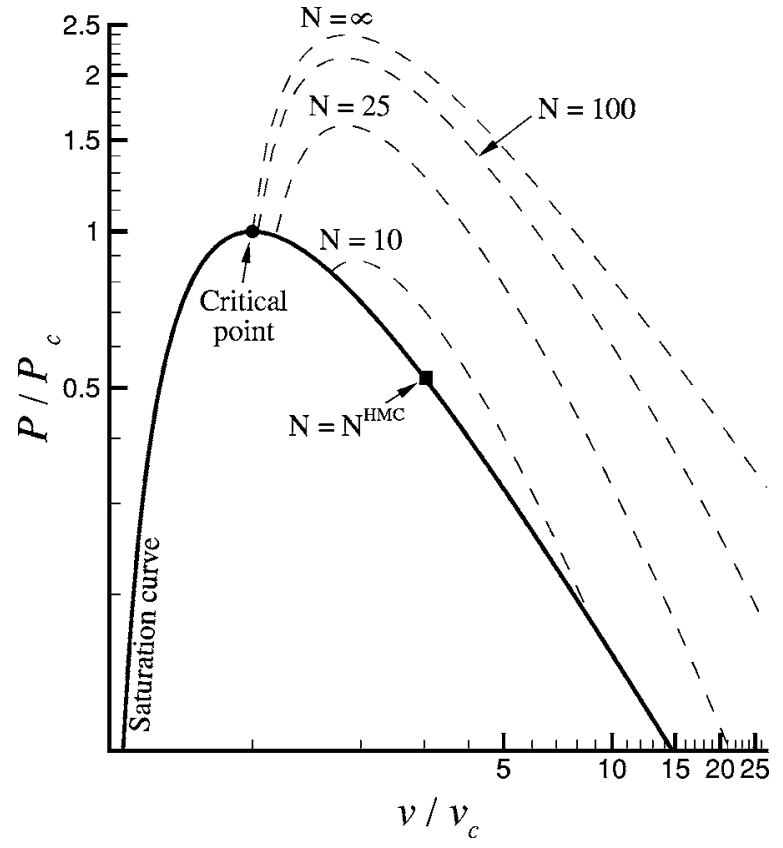

FIG. 13. Dependence of the $\Gamma=0$ curve on $N$. For $N<N^{\text {BZT }}$, the $\Gamma<0$ region is entirely embedded in the region of liquid-vapor equilibrium.

$$
\Gamma\left(T_{r}, v_{r}\right)=\frac{\left(1+\frac{1}{N}\right)\left(1+\frac{2}{N}\right) \frac{3 T_{r} v_{r}}{\left(3 v_{r}-1\right)^{3}}-\frac{1}{v_{r}^{3}}}{\left(1+\frac{2}{N}\right) \frac{T_{r}}{\left(3 v_{r}-1\right)^{2}}-\frac{2}{3} \frac{1}{v_{r}^{3}}},
$$

is reported here for completeness.

As a final remark, the isothermal approximation of a van der Waals fluids turns out to be indeed very favorable as far as nonclassical characteristics are concerned, since it corresponds to the idealized situation $N \rightarrow \infty$. The isothermal van der Waals model can be therefore assumed to feature a simplified system of equations, namely the $P$ system. ${ }^{46}$ It follows that the $P$ system for the isothermal van der Waals gas can be used to study analytical or numerical solutions exhibiting nonclassical features in a conveniently simplified context. In one spatial dimension, the $P$ system is in fact constituted by only two equations (whereas the full Euler equation system has three) and allows for the formation of acoustic waves only, with no contact surfaces.

\section{VAPORS CLASSIFICATION AND WAVE PROPAGATION}

The theoretical developments shown in the preceding sections lead to identify three possible gas dynamic behaviors in isentropic flows for van der Waals fluids depending on the derivative of the speed of sound with respect to the density. More precisely, these different gas dynamic behavior are associated with three different thermodynamic regions which are delimited by the isolines $\Gamma=1$, namely, $\Psi=0$, and $\Gamma=0$. Although the $\Gamma>1$ region exists for all fluids, the $1<\Gamma$ $<0$ and $\Gamma<0$ regions exist in the vapor phase only provided that the fluid is complex enough under a molecular point of view. More precisely, fluids with $N>N^{\mathrm{HMC}} \simeq 7.57$ allow for a $1<\Gamma<0$ region in the vapor phase whereas fluids charac-
TABLE IV. Classification of fluid vapors based on the existence of thermodynamic regions characterized by different behaviors of the speed of sound on isentropic transformations. For polytropic van der Waals fluids the only molecular parameter which determines the fluid class is the molecular complexity given by the number of active degrees of freedom $N$.

Low molecular complexity (LMC) vapors

High molecular complexity (HMC) vapors

Bethe-Zel'dovich-Thompson (BZT) vapors

$$
\begin{gathered}
3<N<N^{\mathrm{HMC}} \\
N^{\mathrm{HMC}}<N<N^{\mathrm{BZT}} \\
N>N^{\mathrm{BZT}}
\end{gathered}
$$

terized by $N>N^{\mathrm{BZT}} \simeq 33.33$ exhibit both the $1<\Gamma<0$ and $\Gamma<0$ regions, in addition to the $\Gamma>1$ that exists for all van der Waals fluids.

Therefore, a classification of fluids (or fluid vapors) can be based on the possibility for a certain fluid to display all three thermodynamic regions, with associated peculiar gas dynamic behaviors, or two or only one. Within the van der Waals approximation, this is solely determined by the molecular complexity $N$. The proposed classification is as follows:

Low molecular complexity (LMC) vapors: Regardless of the value of $N$, all van der Waals vapors allow for the speed of sound to decrease on isothermal compression, in a region located near the liquid-vapor saturation curve. If $N<N^{\mathrm{HMC}}$ $\simeq 7.57$ however the speed of sound can only increase on isentropic compression and decrease on isentropic expansion therefore it changes its value as the wave speed (see Sec. I).

High molecular complexity (HMC) vapors: Vapors characterized by $N>N^{\mathrm{HMC}} \simeq 7.57$, such as, for example, toluene (cf. Table III) display a thermodynamic region in which the speed of sound decreases on isentropic compressions and increases on expansions. The wave speed changes as in the classical case, that is, increases on compressions and decreases on rarefaction, because the modulus of the difference in the speed of sound across the wave, which is opposite in sign to that of the fluid velocity, is lower than that of the fluid velocity itself.

Bethe-Zel'dovich-Thompson (BZT) vapors: If $N>N^{\mathrm{BZT}}$ $\simeq 33.33$, there exists a region near the liquid-vapor saturation curve, where gas dynamic behavior is reversed with respect to the usual behavior encountered in the gas dynamics, viz. gas dynamics of perfect gases. For example, due to the strong decrease in the speed of sound across a compression wave, which is higher in modulus than the increase in the fluid velocity, the wave speed reduces across compression and increases across rarefaction; compression waves spread as isentropic compression fans and rarefaction waves steepen to form rarefaction shocks.

The three fluid families are summarized in Table IV. Note that, even though the information provided by the use of the van der Waals model is not quantitatively accurate, the qualitative conclusions can be held as general.

As an illustration of these concepts, the propagation of a rarefaction wave for three exemplary case is now considered. The model fluid is $\mathrm{D}_{4}\left(N=127.6>N^{\mathrm{BZT}}\right)$ which allows for all possible situations, namely, the LMC, HMC, and BZT behavior under the van der Waal model. They can be observed by suitably selecting the initial and final states across the 


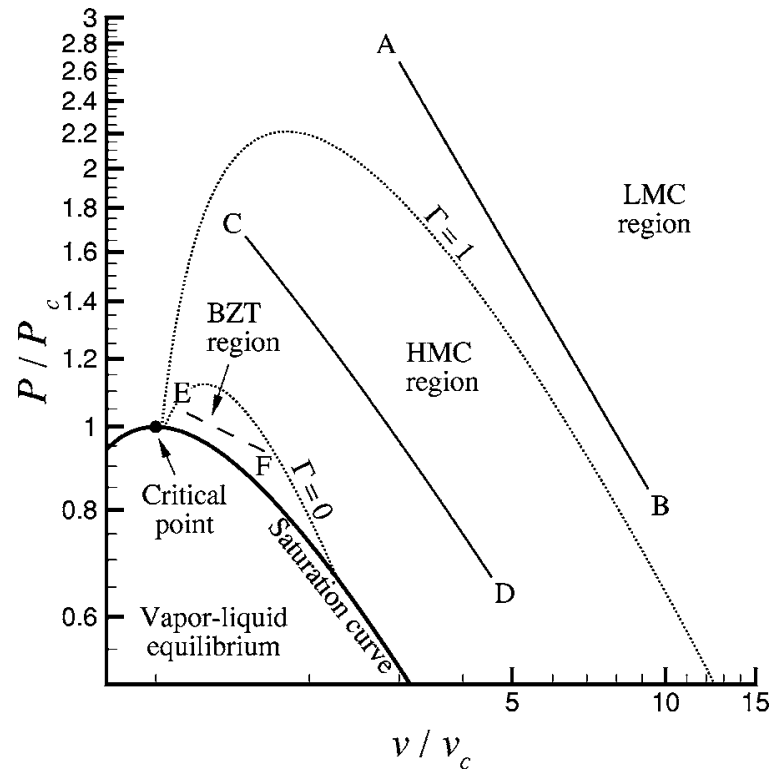

FIG. 14. Exemplary cases of an expansion wave traveling from left to right in a still van der Waals fluid $\left(\mathrm{D}_{4}, N=127.6\right)$. Thermodynamic states in the $P_{r}-v_{r}$ plane relative to the cases presented in Figs. 15-17. Unperturbed states are indicated with $A, C$, and $E$ states past the expansion are indicated with $B$, $D$, and $F$.

rarefaction wave. These are summarized in Fig. 14, where three different rarefaction waves, namely, $A-B, C-D$, and $E-F$, are shown, together with the saturation curve and the $\Gamma=1$ and $\Gamma=0$ boundaries. In all cases a rarefaction wave moves from left to right: the fluid on the right of the perturbation is still (states $A, C$, and $E$ ), while the perturbation puts the fluid (states $B, D$, and $F$ ) into motion in the opposite direction.

Figure 15 shows the solution for the right-running $A-B$ wave in terms of the pressure $P$, the fluid velocity $u$, the

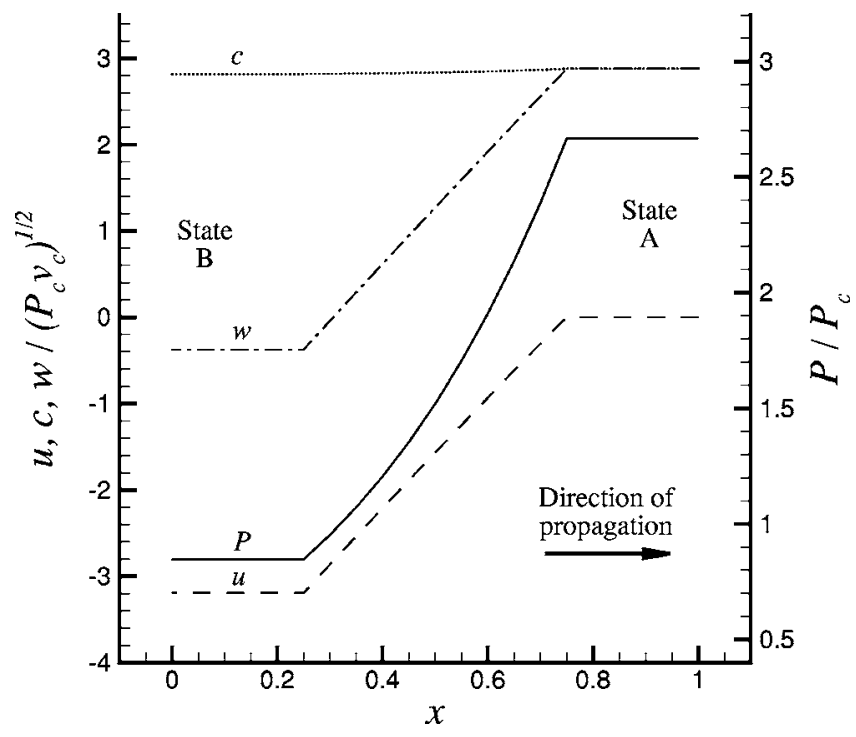

FIG. 15. LMC behavior: isentropic expansion with states belonging to the $\Gamma>1$ region (cf. Fig. 14). The sound speed $c$ slightly decreases past the perturbation therefore the wave speed $w$ also decreases. The velocity of the fluid $u$ and the pressure $P$ are also shown as a function of the spatial coordinate. sound speed $c$, and the wave speed $w=u+c$ profiles. Since $\Gamma>1$, classical LMC behavior is observed, which is qualitative coincident with that of an ideal polytropic gas, with all the considered variables decreasing across the rarefaction wave.

The exact solution to the traveling wave problem has been computed as follows. The Euler equations for compressible nonviscous fluids are first written in the following advection form: ${ }^{36}$

$$
\frac{\partial w}{\partial t}+A(w) \frac{\partial w}{\partial x}=0
$$

with

$$
\boldsymbol{w}=\left(\begin{array}{l}
v \\
u \\
s
\end{array}\right) \quad \text { and } \quad \boldsymbol{A}(\boldsymbol{w})=\left(\begin{array}{ccc}
u & -v & 0 \\
v\left(\frac{\partial P}{\partial v}\right)_{s} & u & v\left(\frac{\partial P}{\partial s}\right)_{v} \\
0 & 0 & u
\end{array}\right) \text {, }
$$

for the primitive variables specific volume $v$, fluid velocity $u$, and specific entropy $s$. The continuous solution across a rarefaction wave from state $\boldsymbol{w}_{0}=\left(v_{0}, u_{0}, s_{0}\right)^{T}$, state $A$ here, is obtained by determining the integral curves ${ }^{47}$ of the hyperbolic system (20), namely, the curves tangent in any state point to the vector field of the eigenvector of $\boldsymbol{A}(\boldsymbol{w})$ associated with the considered wave. In the case under consideration, the rarefaction wave $A-B$ is a 3 -wave and the associated eigenvalue and right eigenvector are $\lambda(\boldsymbol{w})=u+c(s, v)$ and $\boldsymbol{r}(\boldsymbol{w})=(v,-c(s, v), 0)^{T}$, respectively. The integral curve $\boldsymbol{w}(\xi)$ of the 3-wave is therefore obtained as the solution of the following system of ODE:

$$
\frac{d \boldsymbol{w}}{d \xi}=\frac{\boldsymbol{r}(\boldsymbol{w})}{\boldsymbol{r}(\boldsymbol{w}) \cdot \boldsymbol{\nabla} \lambda(\boldsymbol{w})}=\frac{\boldsymbol{r}(\boldsymbol{w})}{c(s, v) \Gamma(s, v)},
$$

with $\boldsymbol{\nabla}=(\partial / \partial v, \partial / \partial u, \partial / \partial s)^{T}$, under the condition $\lambda(\boldsymbol{w}(\xi))=\xi$ and the initial condition $\boldsymbol{w}\left(\xi_{0}\right)=\boldsymbol{w}_{0}$, with $\xi_{0}=\lambda\left(\boldsymbol{w}_{0}\right)=u_{0}$ $+c\left(s_{0}, v_{0}\right)$, which immediately gives $s=s_{0}$ and reduces to the two following systems of ODE:

$$
\begin{aligned}
& \frac{d v}{d \xi}=\frac{v}{c\left(s_{0}, v\right) \Gamma\left(s_{0}, v\right)} \quad \text { with } v\left(\xi_{0}\right)=v_{0}, \\
& \frac{d u}{d \xi}=-\frac{1}{\Gamma\left(s_{0}, v\right)} \quad \text { with } u\left(\xi_{0}\right)=u_{0} .
\end{aligned}
$$

The first equation is separable and can be solved by simple quadrature to give the following implicit definition of the solution $v(\xi)$,

$$
\xi(v)=u_{0}+c\left(s_{0}, v_{0}\right)+\int_{v_{0}}^{v} \frac{c\left(s_{0}, \nu\right)}{\nu} \Gamma\left(s_{0}, \nu\right) d \nu .
$$

Substituting now the function $v(\xi)$ in the ODE for the fluid velocity $u$ and eliminating the variable $\xi$ by introducing the change of variable $\xi \rightarrow v(\xi)$ immediately gives ${ }^{48}$ 


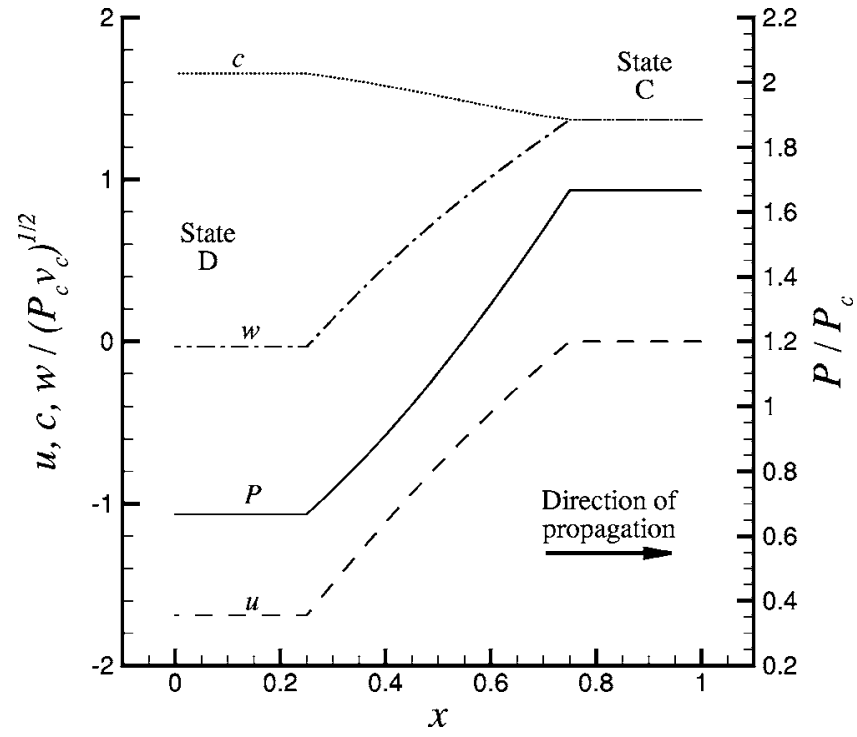

FIG. 16. HMC behavior: isentropic expansion with states enclosed in the region where $0<\Gamma<1$ (cf. Fig. 14). The sound speed increases across the expansion but still the wave speed $w$ decreases.

$$
u(v)=u_{0}-\int_{v_{0}}^{v} \frac{c\left(s_{0}, \nu\right)}{\nu} d \nu
$$

In accordance with the isentropic character of the transformation, the pressure along the rarefaction wave is then computed as

$$
P(v)=\left(P_{0}+\frac{a}{v_{0}^{2}}\right)\left(\frac{v_{0}-b}{v-b}\right)^{1+2 / N}-\frac{a}{v^{2}},
$$

with $p_{0}=P\left(s_{0}, v_{0}\right)$. The solution procedure outlined above requires numerical quadrature to evaluate the integral in Eqs. (23) and (24). Adaptive numerical quadrature has been used here to obtain a relative accuracy lower than $10^{-9}$.

The sound speed along the rarefaction wave is easily obtained by differentiating with respect to $\xi$ the relation $c(\xi)=c\left(s_{0}, v(\xi)\right)$, namely,

$$
\frac{d c}{d \xi}=\left(\frac{\partial c}{\partial v}\right) \frac{d v}{d \xi}=-\frac{\Gamma\left(s_{0}, v(\xi)\right)-1}{\Gamma\left(s_{0}, v(\xi)\right)},
$$

and therefore, substituting again $\xi \rightarrow v(\xi)$,

$$
\frac{d c}{d v}=-\frac{\Gamma\left(s_{0}, v\right)-1}{\Gamma\left(s_{0}, v\right)} .
$$

The relation above clearly shows that the speed of sound decreases along the rarefaction wave provided that $\Gamma>1$, namely, in the $\mathrm{LMC}$ regime.

Conversely, if $0<\Gamma<1$, the speed of sound increases along the rarefaction wave. This is indeed the case of the wave $C-D$, with both states past the perturbation $(D)$ and before the perturbation $(C)$ located in the $0<\Gamma<1$ region (cf. Fig. 14). From Fig. 16, it can be noted that the gas dynamic behavior is still classical $\left(w_{D}<w_{C}\right)$ and the rarefaction wave is a continuous isentropic wave; however the speed of sound decreases across the expansion.

Classical fluid dynamics is reversed in case both the unperturbed state $(E)$ and the state past the expansion $(F)$ are

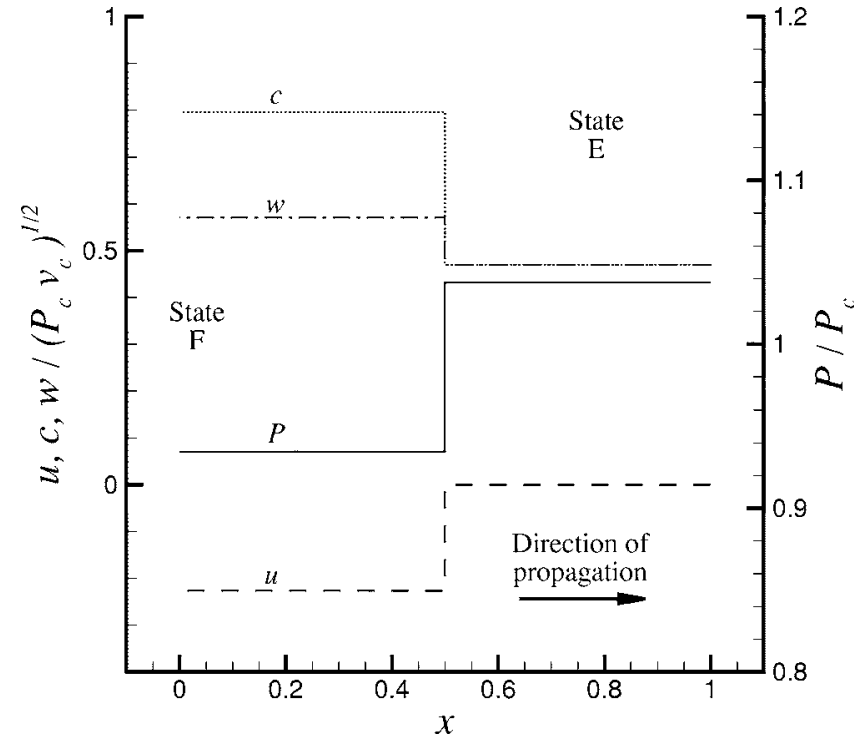

FIG. 17. BZT or nonclassical gas dynamic behavior: the unperturbed state and the state past the perturbation are both inside the negative $\Gamma$ region (cf. Fig. 14). The speed of sound has such an increase across the perturbation that also the wave speed increases and therefore an expansion shock is formed. Also the fluid pressure and velocity are discontinuous across the dissipative $(\Delta s>0)$ expansion.

within the negative $\Gamma$ region (cf. Fig. 14). In this case a rarefaction shock wave is formed and the solution procedure outlined above no longer applies. Instead, the RankineHugoniot jump conditions, namely,

$$
\begin{aligned}
& \Delta(\rho u)=\sigma \Delta \rho, \\
& \Delta\left(\rho u^{2}+P(s, v)\right)=\sigma \Delta(\rho u), \\
& \Delta\left[\rho u\left(h(s, v)+\frac{1}{2} u^{2}\right)\right]=\sigma \Delta\left(\rho e+\frac{1}{2} \rho u^{2}\right),
\end{aligned}
$$

with $\Delta(\cdot)=(\cdot)_{1}-(\cdot)_{0}, \quad \sigma$ shock speed and $h(s, v)=e(s, v)$ $+v P(s, v)$ specific enthalpy, are to be solved to find the postshock state 1 (state $F$ here) from the unperturbed state 0 (state $E$ ). A one-parameter family of solutions is spanned by varying the shock speed $\sigma$. The latter is computed so as to have the desired postshock density $\rho_{1}$ (namely, $\rho_{A}$ ). Figure 17 shows that across the rarefaction shock $E-F$ the increase of the speed of sound across the expansion is so accentuated to more than overcome the decrease of the fluid velocity resulting in the wave speed to increase across the shock wave.

More complicated situations can occur when the initial and final states are located in different thermodynamic regions, say a rarefaction wave from state $A$ to state $F$. In this cases, mixed waves can possibly occurs, including double sonic shocks as described in Ref. 49 . At the $\Gamma=0$ boundary, the characteristic field associated with acoustic disturbances becomes linearly degenerate, $\boldsymbol{r}(\boldsymbol{w}) \cdot \boldsymbol{\nabla} \lambda(\boldsymbol{w})=0$ in Eq. (22), and the technique presented here no longer applies. The solution to this kind of flows requires to resort to the wellknown fully nonlinear theory described, e.g., in Refs. 1 and 
3 , which accounts for the possibility of a wave to cross the $\Gamma=0$ boundary, but is outside the scope of the present analysis.

\section{CONCLUSION}

In this paper the van der Waals' representation of molecular interaction in a vapor is used to explore the relation between molecular characteristics and nonclassical gas dynamic phenomena. The influence of attractive forces, repulsive forces, and molecular complexity (defined as the number of available of molecular degrees of freedom) on the speed of sound is thoroughly analyzed.

Isothermal transformations allow for a direct insight into the role of the mentioned molecular characteristics. In case of complex molecules, isentropic transformations are almost isothermal, therefore the isothermal results can be extrapolated to isentropic flow processes. Gases made of complex molecules have a lower speed of sound and its variation in isentropic transformations in the dense gas region is more accentuated due to the greater role of attraction forces in reducing its value with decreasing density. For these gases therefore it exists a thermodynamic region in which, for instance, the sound speed in an expansion increases more than the velocity of the fluid, allowing for the formation of expansion shocks. A few representative cases of the exact solution of flow field generated by a perturbation traveling in a still fluid are presented to illustrate the influence of all possible variations of the sound speed with density.

Admittedly, the van der Waals model is not accurate for dense gas vapors and it overestimates the value of $\Gamma$, the parameter whose negative values herald the nonclassical behavior. More complex equations of state are currently used to investigate nonclassical gas dynamics phenomena in connection with accurate quantitative empirical data. The use of more sophisticated molecular models would in principle allow for an analysis of the influence of other molecular characteristics like polarity, acentricity, etc., on nonclassical gas dynamic behavior.

It is very important to notice that the thermal stability of complex molecules in practical applications very much limits the existence of thermodynamic states toward high temperatures. As an example the thermal stability limit of $\mathrm{D}_{4}$ in stainless steel has been measured to be above $340{ }^{\circ} \mathrm{C}$ and possibly as high as $400^{\circ} \mathrm{C}$ if appropriately purified, degassed and dehydrated. ${ }^{50}$ Because the critical temperature of $\mathrm{D}_{4}$ is $313.35{ }^{\circ} \mathrm{C}$, its maximum reduced temperature is 1.15 , which clearly shows that the region without a minimum in the sound speed $\left[T_{r} \geqslant(N /(N+2)) \frac{27}{8} \sim 3.32\right.$ or $T \geqslant 1675^{\circ} \mathrm{C}$ for $\mathrm{D}_{4}$ ] can never exist whenever the molecule is a complex organic compound.

${ }^{1}$ R. Menikoff and B. J. Plohr, "The Riemann problem for fluid flow of real material," Rev. Mod. Phys. 61, 75 (1989).

${ }^{2}$ M. S. Cramer, "Nonclassical dynamics of classical gases," in Nonlinear Waves in Real Fluids, edited by A. Kluwick (Springer-Verlag, New York, 1991), pp. 91-145.

${ }^{3}$ A. Kluwick, Handbook of Shock Waves (Academic, New York, 2000), Vol. 1, Chap. 3.4, pp. 339-411.

${ }^{4}$ P. A. Thompson and K. C. Lambrakis, "Negative shock waves," J. Fluid Mech. 60, 187 (1973).
${ }^{5}$ A. A. Borisov, Al. A. Borisov, S. S. Kutateladze, and V. E. Nakaryakov, "Rarefaction shock waves near the critic liquid-vapour point," J. Fluid Mech. 126, 59 (1983).

${ }^{6}$ M. S. Cramer and R. Sen, "Shock formation in fluids having embedded regions of negative nonlinearity," Phys. Fluids 29, 2181 (1986).

${ }^{7}$ M. S. Cramer and R. Sen, "Exact solutions for sonic shocks in van der Waals gases," Phys. Fluids 30, 377 (1987).

${ }^{8}$ B. P. Brown and B. M. Argrow, "Nonclassical dense gas flows for simple geometries," AIAA J. 36, 1842 (1998).

${ }^{9}$ G. H. Schnerr and P. Leidner, "Two-dimensional nozzle flow of dense gases." in Fluids Engineering Conference, No. 93-FE-8, ASME (1993).

${ }^{10} \mathrm{~S}$. H. Morren, "Transonic aerodynamics of dense gases," Technical Memorandum TM 103722, NASA (1991).

${ }^{11}$ M. S. Cramer and G. M. Tarkenton, "Transonic flows of BetheZel'dovich-Thompson fluids," J. Fluid Mech. 240, 197 (1992).

${ }^{12}$ P. Colonna and S. Rebay, "Numerical simulation of dense gas flows on unstructured grids with an implicit high resolution upwind Euler solver," Int. J. Numer. Methods Fluids 46, 735 (2004).

${ }^{13}$ B. P. Brown and B. M. Argrow, "Two-dimensional shock tube flow for dense gases," J. Fluid Mech. 349, 95 (1997).

${ }^{14}$ B. M. Argrow, "Computational analysis of dense gas shock tube flow," Shock Waves 6, 241 (1996).

${ }^{15}$ A. Guardone, "Nonclassical gas dynamics: Thermodynamic modeling and numerical simulation of multidimensional flows of BZT fluids," Ph.D. thesis, Politecnico di Milano, Italy (2001).

${ }^{16}$ G. H. Schnerr and P. Leidner, "Numerical investigation of axial cascades for dense gases," in PICAST'1-Pacific International Conference on Aerospace Science Technology, edited by E. L. Chin, Taiwan, Republic of China, National Cheng Kung University, December 1993, Vol. 2, pp. 818 825.

${ }^{17}$ J. F. Monaco, M. S. Cramer, and L. T. Watson, "Supersonic flows of dense gases in cascade configurations," J. Fluid Mech. 330, 31 (1997).

${ }^{18}$ B. P. Brown and B. M. Argrow, "Application of Bethe-Zel'dovicThompson fluids in organic Rankine cycle engines," J. Power Sources 16, 1118 (2000).

${ }^{19}$ W. D. Hayes, "The basic theory of gas dynamic discontinuities," in High Speed Aerodynamics and Jet Propulsion, edited by H. W. Emmons (Princeton University Press, Princeton, 1960), Vol. 3, pp. 416-481.

${ }^{20} \mathrm{P}$. A. Thompson, "A fundamental derivative in gas dynamics," Phys. Fluids 14, 1843 (1971).

${ }^{21}$ M. H. Zucrow and J. D. Hoffman, Gas Dynamics (Wiley, New York, 1976), Vol. 1.

${ }^{22}$ P. A. Thompson and K. C. Lambrakis, "Negative shock waves," J. Fluid Mech. 60, 187 (1973).

${ }^{23}$ P. A. Thompson, "A fundamental derivative in gas dynamics," Phys. Fluids 14, 1843 (1971).

${ }^{24}$ P. A. Thompson, Compressible Fluid Dynamics (McGraw-Hill, New York, 1988), p. 384.

${ }^{25}$ M. S. Cramer and A. Kluwick, "On the propagation of waves exhibiting both positive and negative nonlinearity," J. Fluid Mech. 142, 9 (1984).

${ }^{26} \mathrm{P}$. A. Thompson and Y. Kim, "Direct observation of shock splitting in a vapor-liquid system," Phys. Fluids 26, 3211 (1983).

${ }^{27}$ P. A. Thompson, G. A. Carofano, and Y. Kim, "Shock waves and phase changes in a large heat capacity fluid emerging from a tube," J. Fluid Mech. 166, 57 (1986).

${ }^{28}$ S. C. Gulen, P. A. Thompson, and H. A. Cho, "Rarefaction and liquefaction shock waves in regular and retrograde fluids with near-critical end states," in Adiabatic Waves in Liquid-Vapor Systems (Springer-Verlag, Berlin, 1989), pp. 281-290.

${ }^{29} \mathrm{P}$. A. Thompson, "Liquid-vapor adiabatic phase changes and related phenomena," in Nonlinear Waves in Real Fluids, edited by A. Kluwick (Springer-Verlag, New York, 1991), pp. 147-213.

${ }^{30}$ J. R. Simões-Moreira and J. E. Shepherd, "Evaporation waves in superheated dodecane," J. Fluid Mech. 382, 63 (1999).

${ }^{31}$ A. G. Ivanov and S. A. Novikov, "Rarefaction shock waves in iron and steel," Zh. Eksp. Teor. Fiz. 40, 1880 (1961).

${ }^{32}$ S. S. Kutateladze, V. E. Nakoryakov, and A. A. Borisov, "Rarefaction waves in liquid and gas-liquid media," Annu. Rev. Fluid Mech. 19, 577 (1987).

${ }^{33}$ M. S. Cramer, "Negative nonlinearity in selected fluorocarbons," Phys. Fluids A 1, 1894 (1989).

${ }^{34}$ S. H. Fergason, T. L. Ho, B. M. Argrow, and G. Emanuel, "Theory for producing a single-phase rarefaction shock wave in a shock tube," J. Fluid Mech. 445, 37 (2001). 
${ }^{35}$ S. H. Fergason, A. Guardone, and B. M. Argrow, "Construction and validation of a dense gas shock tube," J. Thermophys. Heat Transfer 17, 326 (2003).

${ }^{36} \mathrm{H}$. A. Bethe, "The theory of shock waves for an arbitrary equation of state," Technical Report No. 545, Office of Scientific Research and Development (1942).

${ }^{37}$ Y. B. Zel'dovich, "On the possibility of rarefaction shock waves," Zh. Eksp. Teor. Fiz. 4, 363 (1946).

${ }^{38} \mathrm{~K}$. C. Lambrakis and P. A. Thompson, "Existence of real fluids with a negative fundamental derivative," Phys. Fluids 15, 933 (1972).

${ }^{39}$ P. Colonna and P. Silva, "Dense gas thermodynamic properties of single and multicomponent fluids for fluid dynamics simulations," J. Fluids Eng. 125, 414 (2003).

${ }^{40}$ A. Guardone and B. M. Argrow, "Nonclassical gas dynamic region of selected fluorocarbons," Phys. Fluids 17, 116102-1 (2005).

${ }^{41} \mathrm{P}$. Colonna, "Experimental and numerical investigation of dense gas fluid dynamics and BZT fluids exploitation for energy conversion applications," NWO-VIDI project proposal, Delft University of Technology, Delft, February 2004.
${ }^{42}$ D. A. McQuarrie, Statistical Mechanics (Harper-Collins, New York, 1976), Chap. 14-2, pp. 304-306.

${ }^{43}$ A. Guardone, L. Vigevano, and B. M. Argrow, "Assessment of thermodynamic models for dense gas dynamics," Phys. Fluids 16, 3878 (2004).

${ }^{44} \mathrm{H}$. B. Callen, Thermodynamics and an Introduction to Thermostatistics, 2nd ed. (Wiley, New York, 1985).

${ }^{45}$ A. Kluwick, "Internal flows of dense gases," Acta Mech. 169, 123 (2004).

${ }^{46}$ R. J. LeVeque, Numerical Methods for Conservation Laws (Birkhäuser, Basel, 1992).

${ }^{47}$ E. Godlewski and P. A. Raviart, Numerical Approximation of Hyperbolic Systems of Conservation Laws (Springer-Verlag, New York, 1995).

${ }^{48}$ L. Quartapelle, L. Castelletti, A. Guardone, and G. Quaranta, "Solution of the Riemann problem of classical gas dynamics," J. Comput. Phys. 190, $118(2003)$

${ }^{49}$ M. S. Cramer and R. Sen, "Exact solutions for sonic shocks in van der Waal's gases," Phys. Fluids 30, 377 (1987).

${ }^{50}$ P. Colonna Di Paliano, "Fluidi di lavoro multi componenti per cicli termodinamici di potenza," Ph.D. thesis, Politecnico di Milano, Italy (1995). 